Supporting Information

\title{
Micro-pin-finned surfaces with fractal treelike hydrophilic networks for flow boiling enhancement
}

Bo Yuan ${ }^{1}$, Lei Liu ${ }^{1}$, Chenyi Cui ${ }^{1}$, Jiabin Fang 1,*, Yonghai Zhang ${ }^{1}$, Jinjia Wei 1,2,**

${ }^{1}$ School of Chemical Engineering and Technology, Xi' an Jiaotong University, 710049 Xi'an, China

${ }^{2}$ State Key Laboratory of Multiphase Flow in Power Engineering, Xi' an Jiaotong University, $710049 \mathrm{Xi}$ 'an, China

Corresponding author:

* jiabinfang@mail.xjtu.edu.cn and **jjwei@mail.xjtu.edu.cn 


\section{S1. Fractal surface design}

The branch level of paths was defined by a parameter $k$ starting from 0 which represented a single straight path ${ }^{1}$. Based on Murray's law ${ }^{2}$, the design of fractal structure were governed by the following equations:

$$
\begin{gathered}
\gamma=D_{k+1} / D_{k}=2^{-1 / 3} \\
\beta=L_{k+1} / L_{k}=2^{-1 / 2}
\end{gathered}
$$

where $\gamma$ and $\beta$ represented the width and length ratio respectively, as shown in Figure S1.

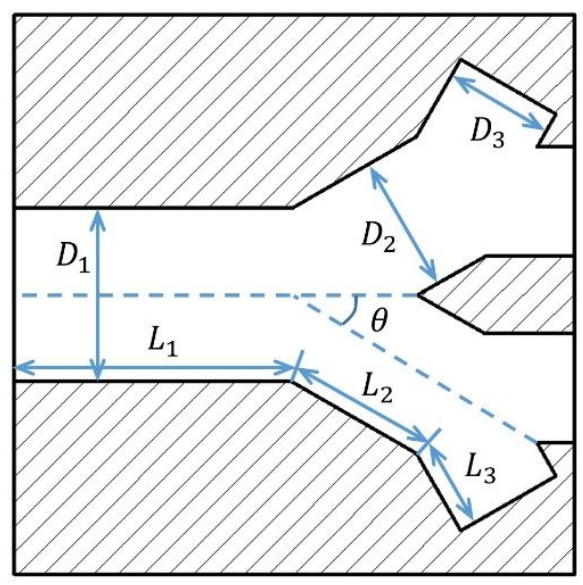

Figure S1 Parameters of the fractal surface

To avoid overlapping of the channels, the bifurcation angle was chosen as $\theta=30^{\circ}$ relative to the line of symmetry. The fraction of the structured covered surface were all kept as $50 \%$ for all the fractal surfaces, so the width and length of each fractal order were different. The dimensions of the first branch level of each fractal surface are listed in Table S1.

Table S1 Dimensions of the fractal surfaces

\begin{tabular}{ccc}
\hline Surface & $D_{1} / \mathrm{mm}$ & $L_{1} / \mathrm{mm}$ \\
\hline F0 & 1 & 10
\end{tabular}




\begin{tabular}{lll} 
F1 & 3.8 & 6.2 \\
F2 & 3.1 & 4.7 \\
F3 & 2.5 & 4.1 \\
F4 & 2.1 & 3.7 \\
\hline
\end{tabular}

\section{S2. Contact angle test}

The working fluid FC-72 is a low surface tension dielectric fluid and it shows super hydrophilic properties on the bare silicon surface as the contact angle test shown in Figure S2. The volume of the droplet in the test was $4 \mu \mathrm{L}$ and the roughness of the bare Si surface was $R a=4.937 \mathrm{e}-1 \mathrm{~nm}$.

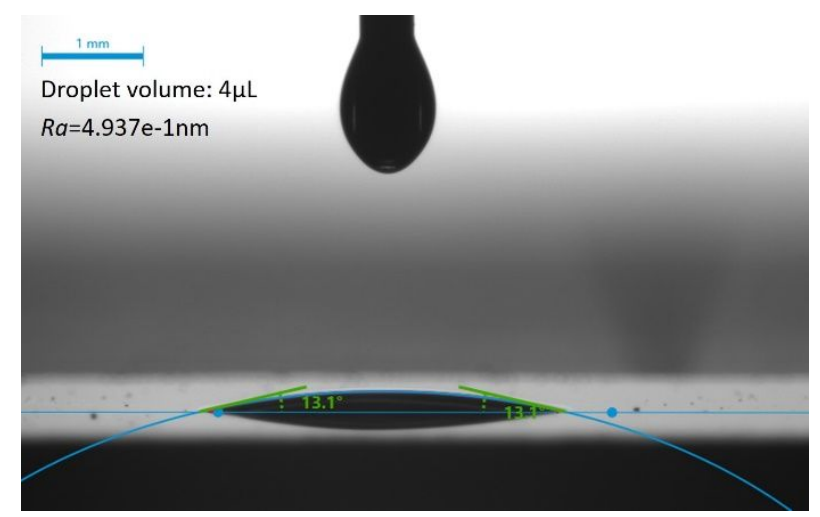

Figure S2 Contact angle test of FC-72 on bare silicon surface

\section{S3. Experimental setup and procedure}

As the sketch map shown in Figure S3, the flow loop used in the experiments included a centrifugal pump, a Coriolis Mass Flowmeter, test section, a liquid tank and the data collection system. The test chip was immobilized on the bottom center of a rectangular flow channel with a cross section dimension of $5 \times 10 \mathrm{~mm}$. For the convenience of observation of bubble behaviors, the flow channel was made by transparent polymethyl methacrylate (PMMA) material. The flow velocity of the 
working fluid FC-72 was measured by a Coriolis Mass Flowmeter and adjusted by the rotating speed of the centrifugal pump. The inlet subcooling of $35 \mathrm{~K}$ was held by a semiconductor cooler. T-type thermocouples were used for the measurement of the test chip $\left(T_{w}\right)$ and bulk fluid $\left(T_{f}\right)$.

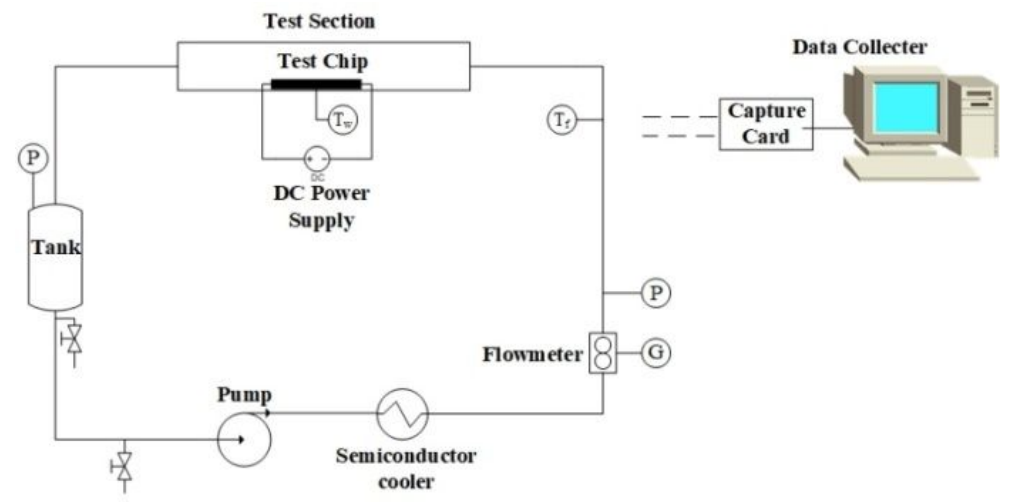

Figure S3 Schematic of the flow loop

The dimension of the silicon chip was $15 \times 10 \times 0.5 \mathrm{~mm}$ with the effective heating area of $10 \times 10 \mathrm{~mm}$. As the schematic of the heating section shown in Figure S4(a), the parts on both sides of the chip were used as electrodes with cooper sires soldered on the bottom for electrical heating. The bottom and side part of the chip were all covered by the low thermal adhesive with thermal conductivity of $0.18 \mathrm{~W} /(\mathrm{m} \cdot \mathrm{K})$ to minimize the heat leak. The whole section was adhered on the PMMA base and sealed into the flow channel. As the fractal structure was asymmetric, the working fluid flows was directional and it flowed from high-level smooth strip to the low-level part as marked in Figure S4(b). 


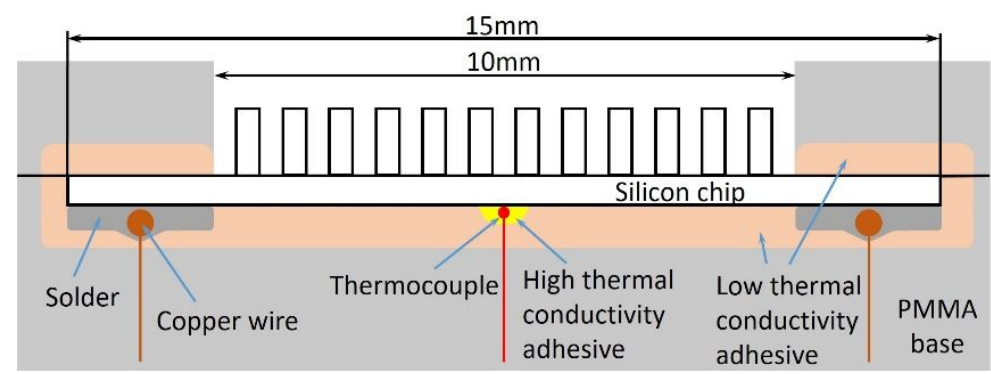

(a) Side view

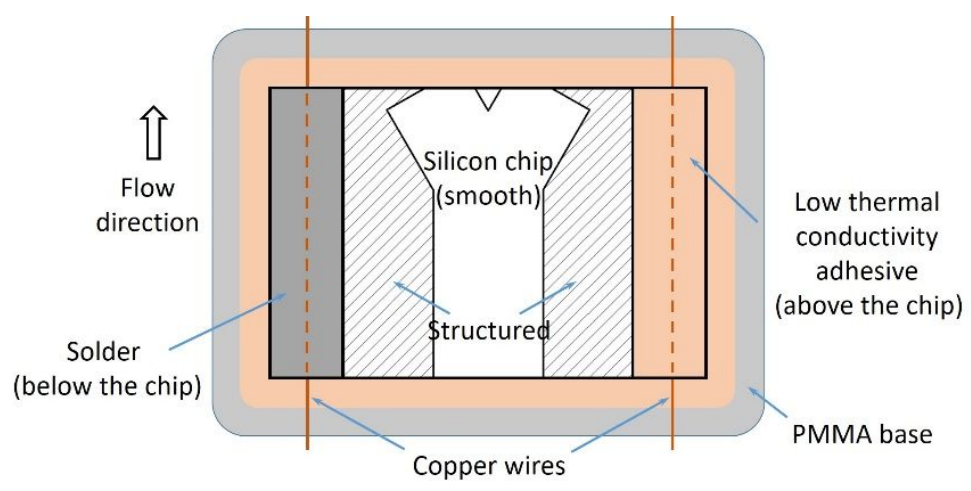

(b) Top view

Figure S4 Schematic of the heating section

During the experiments, the electrical power was added stepwisely, and the data was recorded only if the steady state was reached. The boiling crisis was marked by the sharply rise of surface temperature and the drop of heating current. The power imply should be shut down immediately to protect the equipment.

High-speed camera system was used for the observation of the experimental phenomenon and bubble behaviors from the top side of the flow channel. The photographic system was composed by a high-speed camera (NacMemrecam HX-6E), a micro-lens (NikonAF Micro-Nikkor $60 \mathrm{~mm} \mathrm{f/2.8D)}$ and fill-in light source. The recording rate was $3000 \mathrm{fps}$ and the frame size was $896 \times 688$ pixels. 


\section{S4. Data reduction and uncertainty analysis}

As the silicon chip was directly heated by the electrical power, the heat flux of the chip can be calculated as:

$$
q=\eta V \cdot I / \mathrm{a}^{2}
$$

where $V$ and $I$ are the voltage cross and the current through the chip respectively, and $a$ is the side length of the effective heating area. As the side and bottom of the silicon were all covered by the low thermal adhesive, only small part of the power input was conducted through these parts. The heating efficiency was estimated as $\eta=0.96$ by the simulation by commercial software ANSYS Fluent $18.1^{3}$. The uncertainty of the heat flux was estimated as $0.7 \%$ which included the uncertainties of $V, I$ and $a$ as $0.1 \%$, $0.014 \%$ and $0.5 \%$ respectively.

The heat transfer coefficient was calculated as:

$$
h=\frac{q}{T_{w}-T_{f}}
$$

where $T_{w}$ and $T_{f}$ were the temperatures of the test chip and the bulk fluid measured by thermocouples respectively. The uncertainty of $T_{w}$ was $0.25^{\circ} \mathrm{C}$ which included temperature correction for the measured-value-based surface temperature at the chip bottom $\left(0.2^{\circ} \mathrm{C}\right)$, temperature fluctuations $\left(0.1^{\circ} \mathrm{C}\right)$, and the thermocouple resolution (less than $\left.0.1^{\circ} \mathrm{C}\right){ }^{4}$. The uncertainty of $T_{f}$ was estimated as $0.23^{\circ} \mathrm{C}$ which included the temperature fluctuations of $0.2^{\circ} \mathrm{C}$ and thermocouple resolution of less than $0.1^{\circ} \mathrm{C}$. The uncertainty of the heat transfer coefficient was estimated less than $7.6 \%$ based on the minimum temperature difference of $4.9^{\circ} \mathrm{C}$ at the onset of nucleation boiling. 


\section{S5. Calculation method of heat loss}

The heat loss was estimated by simulation using commercial software ANSYS Fluent 18.1. The geometry of the calculation domain is the same as the polycarbonate base used in the experiment and the boundary conditions are summarized in the following table. The boundary condition of the upward surfaces of the base is convection heat transfer whose heat transfer coefficient is $200 \mathrm{~W} /\left(\mathrm{m}^{2} \cdot \mathrm{K}\right)$ and the free fluid temperature is $21^{\circ} \mathrm{C}(35 \mathrm{~K}$ subcooling of FC-72). The boundary condition of the side and downward surfaces of the base is convection heat transfer whose heat transfer coefficient is $5 \mathrm{~W} /\left(\mathrm{m}^{2} \cdot \mathrm{K}\right)$ and the free fluid temperature is $25^{\circ} \mathrm{C}$ (ambient temperature).

For nucleate boiling with a high heat flux of $20 \mathrm{~W} / \mathrm{cm}^{2}$, the energy source of the silicon chip $(10 \mathrm{~mm} \times 10 \mathrm{~mm} \times 0.5 \mathrm{~mm})$ is $4 \mathrm{e}+8 \mathrm{~W} / \mathrm{m}^{3}$ and the heat transfer coefficient of the top silicon surface is $3458.3 \mathrm{~W} /\left(\mathrm{m}^{2} \cdot \mathrm{K}\right)$ according to the experimental result. The average temperature and the heat flux dissipated from the top silicon surface is $75.7^{\circ} \mathrm{C}$ $(348.8 \mathrm{~K})$ and $19.3 \mathrm{~W} / \mathrm{cm}^{2}$, which corresponds to the heat loss of $4 \%$.

Table S2 Boundary conditions of the simulation

Heat transfer coefficient

Upward surface of the chip

$3458.3 \mathrm{~W} /\left(\mathrm{m}^{2} \cdot \mathrm{K}\right)$

Upward surface of the base $200 \mathrm{~W} /\left(\mathrm{m}^{2} \cdot \mathrm{K}\right)$

Side and downward surface of the base $\quad 5 \mathrm{~W} /\left(\mathrm{m}^{2} \cdot \mathrm{K}\right)$

Temperature

Free fluid

$21^{\circ} \mathrm{C}$

Ambient

$25^{\circ} \mathrm{C}$

Energy source

$4 \mathrm{e}+8 \mathrm{~W} / \mathrm{m}^{3}$ 


\section{S6. Repeatability and hysteresis test}

To test the repeatability of the structured surface, the boiling curves was test for every two days for four times. Taking F2 surface with $U=0.5 \mathrm{~m} / \mathrm{s}$ as an example, as shown in Figure S5, the structured showed good repeatability.

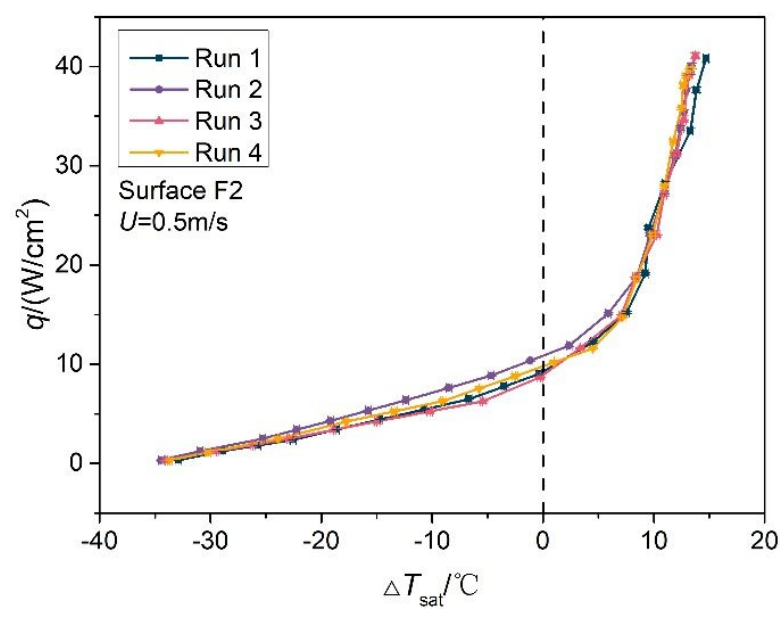

Figure S5 Repeatability test

The results of boiling hysteresis test was shown in Figure S6. The boiling curve of decreasing the heat flux showed slight hysteresis. This was related to the activation of nucleation sites during the boiling process.

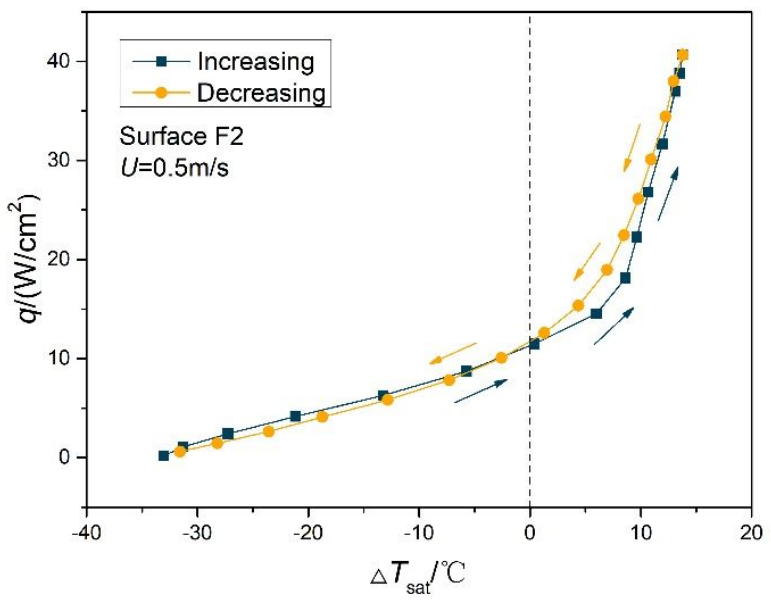

Figure S6 Boiling hysteresis test 


\section{S7. Boiling curves under different flow velocities}

Experiments with different flow velocities from $0.25 \mathrm{~m} / \mathrm{s}$ to $1.0 \mathrm{~m} / \mathrm{s}$ were carried.

The boiling curves under flow velocities of $0.5 \mathrm{~m} / \mathrm{s}, 0.75 \mathrm{~m} / \mathrm{s}$ and $1.0 \mathrm{~m} / \mathrm{s}$ are shown below. The tendency of the boiling curves are similar with that of the flow velocity of $0.25 \mathrm{~m} / \mathrm{s}$ discussed in the manuscript.
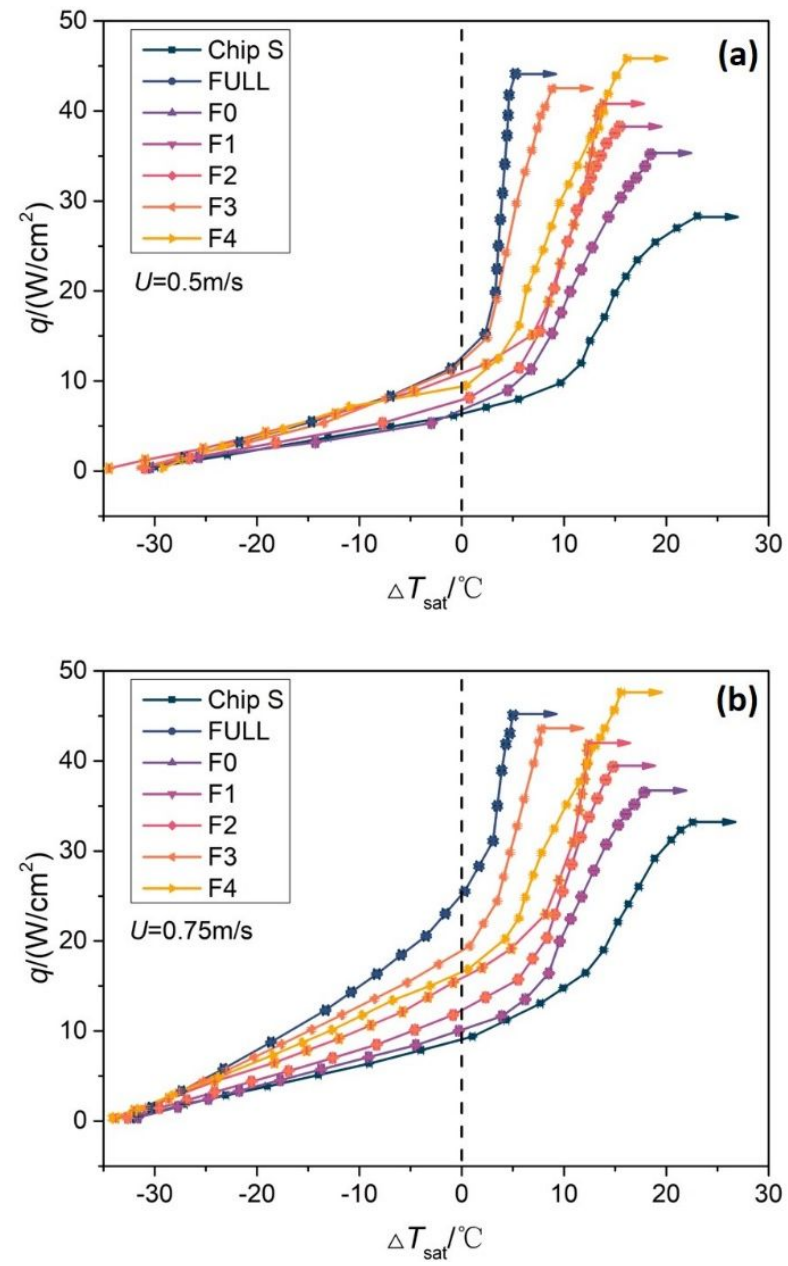


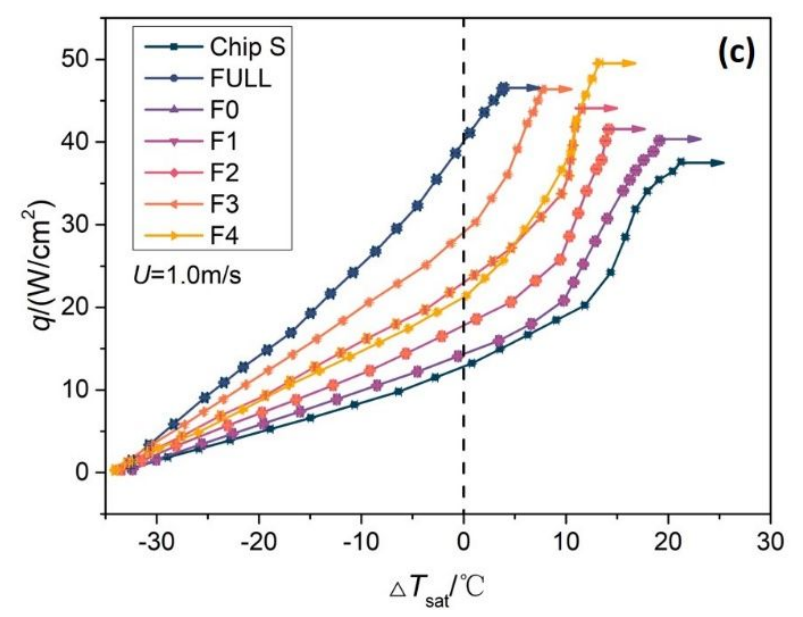

Figure S7 Boiling curves under flow velocities of (a). $U=0.5 \mathrm{~m} / \mathrm{s}$, (b). $U=0.75 \mathrm{~m} / \mathrm{s}$ and (c). $U=1.0 \mathrm{~m} / \mathrm{s}$

\section{S8. The simulation of temperature distribution}

The experimentally tested heat transfer coefficients are used as the boundary conditions. Taking Chip S and FULL, taking $U=0.25 \mathrm{~m} / \mathrm{s}$ and $q=20 \mathrm{~W} / \mathrm{cm}^{2}$ as an example, the comparison of simulated results with the experimental ones are listed in the following table. The temperature difference between the calculated results and the simulated ones are $0.12^{\circ} \mathrm{C}$ and $1.62^{\circ} \mathrm{C}$, which indicates the credibility of the simulation.

Table S3 Comparison of simulated results with experimental ones

\begin{tabular}{cccc}
\hline & Experimental & Simulated & Difference \\
\hline $\begin{array}{c}\text { Smooth surface } \\
\text { Micro-pin-finned } \\
\text { surface }\end{array}$ & $75.61^{\circ} \mathrm{C}$ & $75.73^{\circ} \mathrm{C}$ & $0.12^{\circ} \mathrm{C}$ \\
\hline
\end{tabular}

For fractal surfaces, the higher-order fractal constructions are omitted left only the first order smooth path between the structured parts. Taking $U=0.25 \mathrm{~m} / \mathrm{s}$ and $q=20 \mathrm{~W} / \mathrm{cm}^{2}$ condition as an example, the contour of the simulated temperature distribution is shown in Figure S8 (a). As the structured parts on both sides approach gradually, the relative superheat of the smooth part compared to the structured parts 
decreases and the temperature distribution on the surface of the chip becomes uniform. Figure S8(b) illustrates the variation of relative superheat of the smooth part compared to the structured parts with the width of central smooth path.
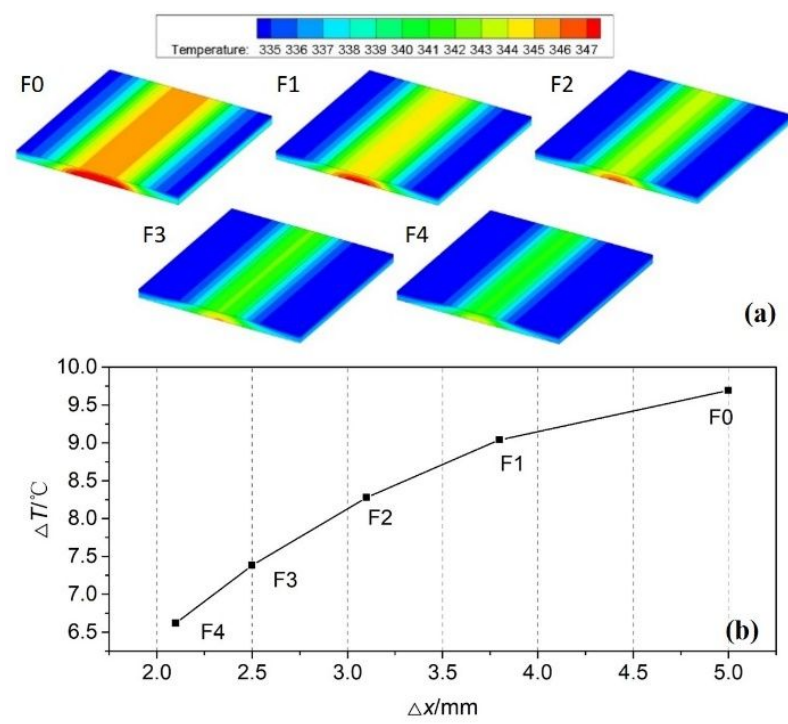

Figure S8 Simulation results of (a) temperature distribution and (b) temperature difference $\left(U=0.25 \mathrm{~m} / \mathrm{s}, q=20 \mathrm{~W} / \mathrm{cm}^{2}\right)$

\section{REFERENCES}

(1) See, Y. S.; Leong, K. C. Experimental study of flow boiling of FC-72 in fractal-like flow channels. International Journal of Thermal Sciences 2019, 140, 184-200.

(2) Murray, C. D. The physiological principle of minimum work-I_the vascular system and the cost of blood volume. Proceedings of the National Academy of Sciences of the United States of America 1926, 12, 207-214.

(3) Yuan, B.; Zhang, Y.; Liu, L.; Wei, J. Flow boiling heat transfer and associated bubble behaviors over backward- and forward-facing steps. Experimental Thermal and Fluid Science 2021, 122, 110300.

(4) Yuan, B.; Zhang, Y.; Liu, L.; Wei, J. Heat transfer enhancement on micro-pin-finned surfaces under high-frequency reciprocating flow. Applied Thermal Engineering 2020, 175, 115378. 